\title{
A Review on Hepatitis B Virus and Hepatocellular Carcinoma
}

\author{
Obeagu Emmanuel Ifeanyi* \\ Department of University Health Services, Michael Okpara University of Agriculture, Nigeria
}

Submission: March 23, 2018; Published: April 19, 2018

"Correspondence Address: Obeagu Emmanuel Ifeanyi, Diagnostic Laboratory Unit, Department of University Health Services, Michael Okpara University of Agriculture, Umudike, Abia State, Nigeria, Tel: +2348037369912; Email: emmanuelobeagu@yahoo.com

\begin{abstract}
Hepatitis B virus is a member of the hepadna virus family, the virus particle consist of outer lipid envelop and an icosahendral nucleocapisd core composed of protein. The nucleus capsid encloses the viral DNA and a DNA polymerase that as reverse transcriptase activity. The genome of hepatitis B virus is made up of circular DNA, but it is unusual because the DNA is not fully double stranded. Transmission of hepatitis B virus results from exposure to infectious blood or body fluids containing blood. Chronic infection with hepatitis B virus may be either a symptomatic or may be associated with chronic inflammation of the liver, leading to cirrhosis which dramatically increases the incidence of hepatocellular carcinoma. It has been irrefutably shown that chronic HEPATITIS B VIRUS infection can lead to hepatocellualr carcinoma (HCC). The most common features of HCC include; abdominal pain, upper abdominal mass, ascites, oedema of the lower extremities, haematemesis, melana and fever, jaundice is a relatively uncommon compliant
\end{abstract}

\section{Introduction}

The earliest record of an epidemic caused by hepatitis B virus was made by Lurman in 1885. An outbreak of small pox occurred in Bremen in (1883) and 1,289 shipyard employers were vaccinated with lymph from other people after several weeks, and up to eight month later, 191 of the vaccinated workers become ill with jaundice and were diagnosed as suffering from serum hepatitis. Other employers who had been inoculated with different batches of lymph remained healthy. Lurman's paper, now regarded as a classical example of an epidemiological study, proved that contaminated hymph was the source of the outbreak.

Later numerous similar outbreaks were reported following the introduction in 1909, of hypodermic needles that were used and more importantly reused, for administering salvarson for the treatment of syphilis. The virus was not discovered until 1965 when Baruch Bhmberg, then working at the NIH (National Institute of Health), discovered the Australia antigen (later known to be hepatitis B surface antigen or HBsAg) in the blood of Australian original people. Although a virus had been suspected since the research published by Maccallum in 1947, while Dane and others discovered the virus particle in 1970 by election microscopy. The virus had been sequenced and the first vaccines were being tested [1-3].

\section{Structure of Hepatitis B Virus}

Hepatitis B virus is a member of the hepadna virus family, the virus particle consist of outer lipid envelop and an icosahendral nucleocapisd core composed of protein [4]. The nucleus capsid encloses the viral DNA and a DNA polymerase that as reverse transcriptase activity. The outer envelope contains embedded proteins which are involved in viral binding of an entry into susceptible cells. The virus is one of the smallest enveloped animal viruses with a viron diameter of $42 \mathrm{~nm}$, but plemorphic forms exist, including filaments and spherical bodies lacking a core. These particles are not infectious and are composed of the lipids and proteins that form part of the surface antigen (HBsAg) and is produced in excess during the life cycle of the virus.

\section{Genome of Hepatitis B Virus}

The genome of hepatitis B virus is made up of circular DNA, but it is unusual because the DNA is not fully double stranded [5]. One end of the full length strand is linked to the virual DNA polymerase. The genome is 3020-3320 nucleotides long (for the full length strand) and 1700-12 2800 nucleotide long (for the short length strand). The negative-sense is complementary to the viral MRNA [6]. The viral DNA is found in the nucleous soon after infection of the cell. The partially double-stranded DNA is rendered fully double stranded by completion of the $(+)$ sense removal of a protein molecule from the (-) sense strand and a short sequence of RNS from the (+) sense strand (Dane, 1970).

Non-coded bases are removed from the ends of the (-) sense strand and the ends are rejoined. There are four known genes encoded by the genome called $\mathrm{C}, \mathrm{X}, \mathrm{P}$ and $\mathrm{S}$. The core protein 
is encoded for by gene (HBsAG) and its start codon is preceded by an upstream in-frame AUG start codon from which the precore protein is produced. HBeAg is produced by proteolytic processing of the pre-core protein [7]. The DNA polymerase is encoded by gene $\mathrm{P}$ gene $\mathrm{S}$ is the gene that codes for the surface (HBsAg). The HBsAg gene is one long open reading frame but contains three in frame start (ATU) codons that divide the gene into three sections; pre-S1, pre-S2 and S Galibert et al. [7]. Because of the multiple start codons, polypeptides of three different sizes called large, middle and small (pre-S1 + pre-S2 + $\mathrm{S}$, pre $-\mathrm{S} 2+\mathrm{S}$, or $\mathrm{S}$ ) are produced. The function of the pre-coded by gene $\mathrm{X}$ is not fully understood [7].

\section{Replication of Hepatitis B Virus (Hepatitis B Virus)}

Hepatitis B is one of the few known non-retroviral viruses which use reverse transcription as part of its replication process [8]. The virus gains entrance into cell by binding to a receptor on the surface of the cell and enters it by endocytosis. Because the virus multiplies through RNS made by a host enzyme, the viral genomic DNA has to be transferred to the cell nucleus by host proteins called chaperones [9]. The partially double-stranded viral DNA is then made fully double-stranded and transformed into covalently closed circular DNA (ccc DNA) that serves as a template for transcription of four viral in RNAS. The largest mRNA, (which is longer than the viral genome), is used to make the copies of the genomes and to make the capside core protein and the viral DNA polymerase [9]. The four viral transcripts undergo additional process and go on to form progeny virons which are released from the cell or return to the nucleus and recycled to produced even more copies. The long mRNA is then transported back to the cytoplasm where the virion protein synthesizes DNA through its reverse transcriptase activity [9].

\section{Serotypes of Hepatitis B Virus (Hepatitis B Virus)}

The hepatitis $\mathrm{B}$ virus serotype are antigenically complex and has specific antigenic determinant referred to as a, d, y, w and $r$. all the serotypes contain a as common sub determinant with pairs of determinant $d$ and $y$; $w$ and $r$ [1]. The four major determinants are therefore; adr, adw, ayr and ayw occur in varying proportions in Northern central and Eastern sub-region of Africa [11]. In the Southern sub-region, nearly all carriers of HEPATITIS B VIRUS serotypes are very helpful epidemiologically, but they have no relationship to the clinical course or outcome of the infection [12].

\section{Genotypes of Hepatitis B Virus}

Genotypes differ by at least $8 \%$ of their sequence and were first reported in 1988 when six were initially described, two further types have since been described ( $G$ and $H$ ) most genotypes are now divided into subgenotypes with distinct properties [13]. Genotype A is most commonly found in the America, Africa, India and Western Europe [14]. Genotype B is most commonly found in Asia and the United States, genotype B1dominate in Japan, B2 is China and Vietnam, while B3 confined to Indonesia B4 is confined to Vietnam [15]. Also these strains specify the serotypes Aywi, BS is most common in the Philippines, genotype $\mathrm{C}$ is most common in Asia and the United States, subgenotype C1 is common in Japan, Korea and china, C2 is common in China, South-East Asia and Bangladesh and C3 in oceania, all this strains specify the serotype adrq [15].

C4 specifying ayw3 is found in Aborigines from Australia, genotype D is commonly found in Southern Europe, India and the United States and has been divided into 8 subtypes (D1-D8) in Turkey genotype $\mathrm{D}$ is also the most common type A pattern of defined geographical distribution is less evident with D1-D4 where these sub genotypes are widely spread within Europe, Africa, and Asia, this may be due to their divergence having occurred before than of genotypes B and C [15]. D4 appears to be the oldest split and is still the dominating sub genotype of D in oceanic. Type E is most commonly found in West and South Africa, Type F is most commonly found in central and South America two subgroups (F1 and F2) genotypes G has an insertion of 36 nucleotides in France and the United States, Type $\mathrm{H}$ is most commonly found in central and South America and California in United States [15].

Africa has five genotypes (A-E) of these the predominant genotypes are A in Kenya, B and D in Egypt, D in Tunisia, A-D in South Africa and E in Nigeria [15].

\section{Epidemiology of Hepatitis B Virus}

The primary method of transmission reflects the prevalence of chronic hepatitis B virus infection in a given area [7]. In low prevalence areas such as the continental United States and Western Europe, where less than $2 \%$ of the population is chronically infected, injection, drug abuse and unprotected sex are the primary factors, although other factors may be important [15]. In moderate prevalence areas, which includes; Eastern Europe, Russia, and Japan were $2-7 \%$ of the population is chronically infected; the disease is predominately spread among children. In high prevalence areas such as China and South East Asia, transmission during child birth is most common, although in other areas of high endemicity such as Africa, transmission during childhood is a significant factor. The prevalence of chronic hepatitis B virus infection in areas of high-endemicity is at least 8\%-20\% [16]. According to WHO report about 2 billion people worldwide have been infected with hepatitis $B$ virus and about 350 million live with chronic infection, an estimated 600, 000 persons die each year due to the acute or chronic cases [17].

Hepatitis B is endemic in China and other parts of Asia, most people in the region become infected with hepatitis $B$ virus during childhood. In these regions, $8 \%$ to $10 \%$ of the adult populations are chronically infected. High rates of chronic hepatitis B virus infections are also found in the Amazon and the southern parts of Eastern and central Europe. In the Middle East and Indian sub-continent, an estimated $2 \%$ to $5 \%$ of the general population is chronically infected, less than $1 \%$ of the 
population in Western Europe and North American is chronically infection [18]. In Nigeria, hepatitis B virus infection has reached hyperendemic levels with the sero-prevalence of $\mathrm{HBaAg}$ estimated to range from $10 \%-40 \%$ despite the availability of a safe and effective vaccine since 1982 and it inclusion in Nigeria's National Immunization program in 1995 the vaccine became available to the country only in 2004 , owing to this late entry and the absence of a national hepatitis B virus surveillance program, the burden of hepatitis B remains substantial it is also believed that every 1 in 4 Nigerian have hepatitis B virus marker [15].

\section{Incubation Period}

The period between transmission of the hepatitis B virus and the beginning of symptoms of hepatitis B which is known as hepatitis B incubation period last between 30 and 180 days. The length of this incubation period will depend on how many virus particles a person have been exposed to [19].

\section{Transmission of Hepatitis B Virus}

Transmission of hepatitis B virus results from exposure to infectious blood or body fluids containing blood. Possible forms of transmission include (but are not limited to) unprotected sexual contact, blood transfusions, reused of contaminated needles and syringes, and vertical transmission from mother to child during childbirth. Without intervention, a mother who is positive for HBsAg confers a $20 \%$ risk of passing the infection to her offspring at the time of birth [20]. The risk is as high as $90 \%$ if the mother is also positive for HBeAg. Hepatitis B virus can be transmitted between family members within households, possibly by contact of non-intact skin or mucous membrane with secretions or saliva containing hepatitis B virus. However, about $30 \%$ of reported Hepatitis B among adult cannot be associated with an identifiable risk factor [20].

Other risk factors for developing hepatitis B virus infection include working in a health care setting, transfusions and dialysis, acupuncture, tattooing, extended overseas travel and residence in a research institution [20].

\section{Pathogenesis of Hepatitis B Virus}

Cirrhosis of the liver which is liver cancer may result from hepatitis B. the hepatitis B virus primarily interferes with the functions of the liver by replicating liver hepatocytes [21]. The receptors is not yet known, though there is evidence that the receptor in the duck hepatitis B virus is carboxypeptidase hepatitis B virus virions binds to the host cell through the pre $S$ domain of the viral surface antigen and are subsequently internalized by endocytosis [21]. Pre S and IgA receptors are accused of these interactions. Hepatitis B virus-pre S specific receptors are primarily expressed on hepatocyte; however, viral DNA and proteins have also been detected in extra hepatic cells.

During Hepatitis B virus infection, the host immune responses cause both hepatocellular damage and viral clearance. Although the innate immunity does not play a significant role in these processes, the adaptive immune response, particularly virus-specific cytotoxic T-Lymphocytes (CTLs), contributes to most of liner injury associated with hepatitis B virus infection [22]. By killing infected cells and by producing antiviral cytokines capable of purging hepatitis B virus from viable hepatocyte, (TLS eliminate the virus, although liner damage is initiated and mediated by the CTLs, antigen non specified inflammatory cells can worsen CTL-induced immunopathology and platelets activated at the sites of infection may facilitate the accumulation of CTLS in the liver hepatocellular carcinoma is also a life threatening issue that can result from chronic hepatitis $\mathrm{V}$ viral infection [22].

\section{Hepatitis B Virus With Acute Hepatitis}

When an individual is infected with hepatitis B virus, an acute stage of the infection is reached. This stage can recovered or lead to fulminant hepatitis, which eventually lead to death [23]. Macroscopic appearances of the liver are slightly enlarged and more or less green depending on the phase of acute disease and the level of jaundice [24]. Acute infection with hepatitis B virus is also associated with general health, loss of appetite, nausea, vomiting, body aches, mild fever and dark urine [16]. Microscopically, according to Kerkar [25], the major findings include

i. Diffuse liver cell injury with lobular disarray.

ii. Necrosis of random, isolated liver cells or small cluster of cell.

iii. Reactive changes of kuffer cells and sinusoidal living cells.

iv. An inflammatory infiltrate in the portal tracts.

v. Evidence of hepatitis regeneration during the recovery phase.

\section{Hepatitis B Virus With Chronic Hepatitis}

Chronic infection with hepatitis B virus may be either a symptomatic or may be associated with chronic inflammation of the liver, leading to cirrhosis which dramatically increases the incidence of hepatocellular carcinoma ( $\mathrm{Hcl})$. Hepatitis $B$ virus has been linked to the development of membranous glomerulonephritis (MGN) [26]. Approximately, 10\% of patient who develop hepatitis B virus becomes chronic carriers of HBsAg of these, about 70\% develop chronic persistent hepatitis (CPH) and the remainder (or 30\% of the total) has chronic acute hepatitis (CAH). Chronic persistent hepatitis ( $\mathrm{CPH}$ ) when associated with hepatitis B virus is usually a benign disease and patients are generally in good health mild hepatomegally is common but usually no progression to cirrhosis is seen [27].

Laboratory abnormalities are episodic elevation of transaminase levels without jaundice. Chronic acute hepatitis $(\mathrm{CAH})$ is frequently a silent disease with minimal correlation between symptoms and liver damage [27]. In about $50 \%$ of 
patients the chronic presentation is jaundice, portal hypertention and ascitis all of which are late manifestation of liver disease [28]. CAH is more likely to progress in males and patients who present at more than 40 years of age [27]. Sometimes apparently stable CAH may have a relapse which can be fatal. This can occur spontaneously but is more often associated with withdrawal of corticosteroid or immunisuppressive drugs [14]. It appears that viral replication occurs during periods of immunosuppression which courses liver injury. A disordered immune response is able to underline the development of $\mathrm{CAH}$. It has been postulated that lack of human leukocytes antigen (HLA) type II and HBcAg expression, together with poor cytotoxic T-cell function may prevent the clearance of hepatitis B virus-infected hepatitis [27].

\section{Hepatitis B Virus among Children and Infants}

Most deaths due to hepatitis B virus occurs in adults, almost 95\% from chronic liver disease such as liver cirrhosis and hepatocellular carcinoma and the remaining $5 \%$ from fulminant hepatitis, the main risk factor for chronic hepatitis B virus related liver disease is chronic infection acquired at birth or in primary infection is asymptomatic, treatment has a limited impact on the pool of individuals with asymptomatic persistent chronic hepatitis B virus infection or symptomatic hepatitis B virus related liver disease [20]. Coupled to this, the long time lag between acquiring the infection and developing the disease means that the strategy of infant vaccination will have little impact on the incidence of liver cirrhosis or cancer for the next 20-30 years [28].

Monitoring the occurrence of acute hepatitis B will also be limited value in assessing a strategy aimed at controlling the acquisition of chronic infection during childhood as acute disease occurs, mainly in older children, which serves as proxy for chronic hepatitis B virus infection, is the first indicator that will be affected by an infant vaccination program and can, therefore, be used to monitor the program's impact over the short term [29].

\section{Test for Children At Least 5 Years of Age}

Persistent chronic hepatitis B virus infection mainly results from either vertical transmission at birth or horizontal transmission in children aged less than 5 years, the chances for a child developing a chronic hepatitis B virus infection is $90 \%$ if infected at birth, 30\% if infected between 1 and 5 years of age and only $5-10 \%$ if infected, after the age of 5years [18]. Moreover, it has been estimated that almost three-quarters of all hepatitis B virus related deaths occur in individuals infected before the age of 5years, in the prevaccination era, the seroprevalence of HBsAg increased little after childhood in most countries, a national survey conducted in 1979-1980 in China, for example, report on HBsAg seroprevalence of $3.2 \%$ in children aged less than 1year, which increased rapidly to 8.9\% 1-4 years old children because of the vaccination program [30]. Hepatitis $B$ virus infection occurs in newborn, infant and children and frequently unrecognized.
Symptoms of hepatitis B virus among children, Newborn and Infant, deep jaundice, bleeding and seizures, unconsciousness are common symptoms.

a. The major route of infection of hepatitis B virus among children or childhood is transplacental infection transmitted to the baby across the placental by mother who is a chronic carrier, almost all children infected in this way become chronic carriers themselves.

b. Another major route is blood transfusion,

c. The rare routes are injection with unclean syringes, organ transplantation and sexual abuse.

\section{Laboratory Diagnosis}

The diagnosis method of hepatitis B virus infection can be grouped as follows:

\section{Isolation and identification of the organisms}

Although numerous cell systems have been used, hepatitis B virus has been reported to have been cultured in Lymphoblast, but this has not become a widely utilized means of studying the virus [29]. The virus will only replicate efficiently in humans and chimpanzees, although other non-human primates have been found to be positive for hepatitis B virus markers [29]. Inspite of the inability to culture hepatitis B virus and of the limited range of susceptible hosts, hepatitis B virus has been extensively characterized because of the ease with which the virus is purified from either blood or liver tissue of infected humans and chimpanzees. Extensive characterization of the virus has led to the identification of a number of antigen and widely used in identifying various stages of the natural course of hepatitis B infection [29]. In addition, the characterization and elucidation of the organization of hepatitis B virus genome have resulted in the development of nucleic and hybridization probes that have been used to identify hepatitis B virus DNA in serum as well as in tissue and expression of various antigens by recombinant DNA technology.

\section{Serological and Immunological diagnostic methods}

The presence of HBsAg in the serum of individuals indicates acute or chronic hepatitis B virus infection [29]. Test for HBsAg has result in the insensitive immunodiffusion methods to the most sensitive third-generation test configured either as reserved passive haemagglutination assays (RPHA), Radioimmunoassay or enzyme linked immunoassays (EIA) currently RIA and EIA are the most widely used methods to detect HBsAg [18].

The monoclonal antibodies to HBsAg have been produced and there have been reports of improved test sensitivity using these probes. A serum sample positive for HBsAg indicates hepatitis $B$ virus infection and it does not fully determine the stage of the disease without additional clinical information such as duration of test positively virion or determine the potential infectivity of the patient. An individual is classified as a chronic 


\section{Cancer Therapy \& Oncology International Journal}

carrier of hepatitis B virus if HBsAG remains positive for more than six month [18].

All HBsAg-positive people should be considered infectious however; the relative degree of infectivity can be assessed by the presence of other marker such as HBsAg or hepatitis B virus DNA. Several types of HBsAg exist and reagents for sub-typing of HBsAg has proven useful in selected investigation of disease transmission but is not routinely used for diagnostic purposes [18].

\section{Treatment of Hepatitis B Virus Infection}

Acute hepatitis B infection does not usually require treatment because most adults clear the infection spontaneously [30]. Early antiviral treatment may only be required in less than $1 \%$ of patients, whose infection takes a very aggressive course (fulminant hepatitis) or who are immunocompromised. On the other hand, treatment of chronic infection may be necessary to reduce the risk of cirrhosis and liver cancer. Chronically infected individuals with persistently elevated serum alanine aminotransferase, a marker of liver damage, and hepatitis B virus DNA levels are candidates for therapy [30]. Although none of the available drugs can clear the infection, they stop the virus from replicating and minimizing liver damage such as cirrhosis and liver cancer. Currently, there are about five to seven medications licensed for treatment of hepatitis B virus infection. These include antiviral drugs lamivudine, Adefovir, Tenofovir, Telbivudine and entecavir and the two immune system modulators interferon alpha-2a.

The use of interfere on, which requires injections daily or three times weekly has been supplanted by long acting pegylated interferon, which is injected only once weekly. However, since individual are much likely to respond than others and this might be because of the genotype of the infection or the patients heredity [30]. The treatment works by the reduction of viral load which in turn reduce viral replication in the liver.

Infant born from mothers known to carry hepatitis B virus can be treated with antibody to the hepatitis B virus (hepatitis B immune globulin or HBIG) when given with the vaccine within twelve hours of birth, the risk of acquiring hepatitis B virus is reduced to $95 \%$. This treatment allows a mother to safely breastfed her child [30].

\section{Reactivation of Hepatitis B Virus}

Hepatitis B virus DNA persists in the body after infection and in some people the disease reoccurs. Although rare, reactivation is seen, most often in people with impaired immunity, hepatitis B virus goes through cycles of replication and non-replication [30]. Approximately $50 \%$ of patients experience acute reactivation. Male patients with baseline ALT of $200 \mu \mathrm{l} / \mathrm{L}$ are three times more likely to develop a reactivation than patients who undo chemotherapy are at risk for hepatitis B virus reactivation. The current view is that immunosuppressive drugs for and increased hepatitis B virus replication while inhibiting cytotoxic $\mathrm{T}$ cell function in the liver [30].

\section{Prevention and Control}

Several vaccines have been developed for the prevention of hepatitis B virus infection. These rely in the use of one of the viral envelope protein (hepatitis B surface antigen or HBsAg). The vaccine was originally prepared from plasma obtained from patients who had long standing hepatitis B virus infection. However, these are more often made using recombinant DNA technology, though plasma derived vaccines continue to be used; the two types of vaccines are equally effected and safe. Following vaccination, HBsAg may be detected in serum for several days; this is known as vaccine antigenaemia [28]. Vaccine is generally administered in two, three or four dose schedules and can be received for $85 \%$ of individuals and can least for 23 years unlike hepatitis A, hepatitis B does not generally spread through water and food. Instead, it is transmitted through body fluids from which prevention is taken to avoid; unprotected sexual contact, blood transfusion, used of contaminated needles and syringes, and vertical transmission during childbirth, infant by be vaccinated at birth [27].

\section{Hepatitis B Virus With Hepatocellular Carcinoma}

It has been irrefutably shown that chronic HEPATITIS B VIRUS infection can lead to hepatocellualr carcinoma (HCC). In Taiwan and South Africa, $60-80 \%$ of patient with HCC are HBsAg positive as compared to approximately $10 \%$ of the general population. The most common features of HCC include; abdominal pain, upper abdominal mass, ascites, oedema of the lower extremities, haematemesis, melana and fever, jaundice is a relatively uncommon compliant [31]. Various preneplastic syndromes such as hypoglycaemia, hypercalcaemia, erythrocytosis, and hypercholesterolaemia can confound the diagnosis, this making the example of internt tumour.

Macroscopically, liver cancer appear as

i. A single large tunor or

ii. Multiple tunours

iii. Poorly define tunor with an infiltrative growth pattern.

Microscopically, there are four architectural and cytological types (pattern) of HCC; filrrolamella, pseduoglandular (adenoid), pleomorphic (giant cell) AND clear cell. In well differentiated forms turnour cells resemble hepatocytes, form trabeculae, cords, and nest, and many contain bile pigment in the cytoplasm in poorly differentiated forms, malignant epithelial cells are discohessive, pleomorphic, anaplastic and giant. The tunor has a scount stroma and central necrosis because of poor visualization.

\section{Conclusion}

Hepatitis B virus is a member of the hepadna virus family, the virus particle consist of outer lipid envelop and an icosahendral nucleocapisd core composed of protein. Chronic 
infection with hepatitis B virus may be either a symptomatic or may be associated with chronic inflammation of the liver, leading to cirrhosis which dramatically increases the incidence of hepatocellular carcinoma. It has been irrefutably shown that chronic hepatitis B virus infection can lead to hepatocellualr carcinoma (HCC).

\section{References}

1. Obeagu EI, Obeagu GU (2017) Hepatitis B Virus and Immunity. Academic Journal of Life Sciences 3(7): 36-46.

2. Obeagu EI, Obeagu GU, Nwosu DC (2016) Hepatitis B and Hepatitis C viral infection: A Review. International Journal of Current Research in Chemistry and Pharmaceutical Sciences 3(11): 10-21.

3. Obeagu EF, Onyenweaku FC, Nwobodo HA, Ochei KC, Ochiabuto Ogochukwu MTB, et al. (2017) Impact of HIV and Hepatitis B Virus Coinfection on Selected Haematological Markers of the Patients in Umuahia, Abia State, Nigeria. Ann Clin Lab Res 5: 2.

4. Pramoolsinsup C (2002) Management of viral hepatitis B. Journal of gastroenterology and hepatology 17(suppl): S512-S545.

5. Magnius LO, Norder (1995) Subtypes, genotypes and molecular epidemiology of the hepatitis $\mathrm{V}$ virus as reflected by sequence intervirology. 38(1-2): 24-34.

6. MacCallum FO (1947) Homologous Serum Hepatitis. Lancet 2: 691.

7. Galibert FJ, Mandert N, Fitoussi B, Toillais I, Charnay V (1979) Nucleotide sequence of the hepatitis B virus genome (subtype ayw) cloned in E. coli. Nature 281: 646.

8. Zuckerman J (2006) Vaccination against hepatitis A and B: Current Opinion in infectious disease 19(5): 456-459.

9. Locarnin S (2004) Molecular Virology of Hepatitis. B. Virus. Seminars in Liver Disease 24(1): 3-10.

10. Kay A, Zoulum F (2007) Hepatitis B virus genetic variability and evolution. Virus Res 127 (2): 164-176.

11. Beck J, Nassal G (2007) Hepatitis B virus replication. World Journal of gastroenterology 13(1): 48-64.

12. Bouchard M, Schrieder R (2004) The enigmas gene of hepatitis B. virus morphogenesis. World Journal of gastroenterology 13(1): 65-73.

13. Howard CR (2006) The biology of hepadnaviruses. J Gen Virol 67(7): 12-15.

14. Bell SJ, Nyuyen T (2009) The management of hepatitis B. Aust presser 23(4): 99-104.

15. Custer B, Sullivan SD, Hazlet TK, Iloeje U, Veenstra DL, et al. (2004)
Global epidemiology of Hepatitis B virus. J Clin Gastroenterol 38(10): 515-868.

16. Redd JT, Baumbac J, John W, Naman O, Khristoua M, et al. (2007) Patient to patient transmission of hepatitis B virus associated with oral surgery. J Infect Dis 195(9): 131-134.

17. Alter MJ (2003) Epidemiology and Prevention Hepatitis B. Semin Liver Dis 23(1): 39-46.

18. Barker LF (1996) Transmission of serum hepatitis 1970. JAMA 276(10): 841-844.

19. Shapino CN (1993) Epidemiology of hepatitis B. The Pediatric infectious disease journal 12(5): 43-37.

20. Glebe D, Urban T (2007) Viral and cellular determinants involved in hepadna viral entry. World J Gastroenterol 13(1): 22-38.

21. Iannacone M, Sitia G, Isogawa M, Marchese P, Castro MG, et al. (2007) Platelets mediate cytotoxic T. hymphocyte indicued liver damage. Nature medicine 11(11): 1167-1169.

22. Ng F, Cohang M, Chan Cheng C, Leung H, Cheng N, et al. (2005) Host heterogeneous ribonucleoprotein $\mathrm{K}(\mathrm{hnRNK})$ as a potential target to suppress hepatitis B-replication PLOS medicine 2(7): 163.

23. Kramvis A, Kew M, Francis G (2005) Hepatitis B virus genotypes. Vaccine 23(19): 2409-2423.

24. Lai CL, Vuen D (2007) The natural history and treatment of chronic hepatitis B: a critical evaluation of standard treatment criteria and points 3(2): 58-61.

25. Kerkar N (2005) Hepatitis B in children: complexities in management. Pediatric transplantation 9(5): 685-691.

26. Kats L, Fraser A, Gafter-Gvilli A, Leiboviu E, Turkaspa R (2008) Launiviudine presents reactivation of hepatitis $B$ and reduces mortality in immuno suppressed patients: Systematic review and meta-analysis. J Viral Hepat 15(2): 89-102.

27. Chu CM, Liaw YF (2007) Prediction factors for reactivation of hepatitis B following hepatitis B antigen seroconversion in chronic hepatitis B. Gastroenterology 133(5): 1458-1465.

28. Chang M (2007) Hepatitis B virus infection. Seminars in fetal and neonatal medicine 12(3): 160-167.

29. Shuping T, Jisu L, Jack RW (1999) Carboxypeptidase D is an avian hepatitis B virus receptor. J Virol 73(10): 8696-8702.

30. Taylor JM (2006) Hepatitis Delta virus. Virology 344(1): 71-76.

31. Lui AU, Li K, Tam R, Tong J, Lai O (1991) Membranous nephropathy related HEPATITIS B VIRUS in adults. The New England Journal of medicine 324(21): 145-763.

This work is licensed under Creative

Commons Attribution 4.0 License

DOI: 10.19080/CTOIJ.2018.10.555784

\section{Your next submission with Juniper Publishers} will reach you the below assets

- Quality Editorial service

- Swift Peer Review

- Reprints availability

- E-prints Service

- Manuscript Podcast for convenient understanding

- Global attainment for your research

- Manuscript accessibility in different formats ( Pdf, E-pub, Full Text, Audio)

- Unceasing customer service

Track the below URL for one-step submission https://juniperpublishers.com/online-submission.php 